\title{
THE GALACTIC DISTRIBUTION AND SUBTYPE EVOLUTION OF WOLF-RAYET STARS
}

\author{
B. Hidayat 1 , A. G. Admiranto 1, K. R. Supelli2 and K. A. van der Hucht ${ }^{3}$ \\ 1 Observatorium Bosscha. ITB. Lembang. West Java, Indonesia \\ 2 Ministery of Science and Technology. BBPT, Jakarta. Indonesia \\ 3 SRON Space Research Laboratory. Utrecht. The Netherlands
}

\begin{abstract}
The galactic distribution of Wolf-Rayet stars is redetermined. In the solar neighbourhood within $d<2.5 \mathrm{kpc}$ the WN/WC number ratio is 0.54 , indicating a larger influence of mass loss and convective overshooting than most present evolutionary models account for. The WR density gradient in the solar neighbourhood is constant. From a comparison of WR galactocentric distances and WR cluster and association membership. we find indications that WCE stars descend from WNL stars at $A>6.5$ kpc. and that WCL stars descend from WNL stars at $R<8.5 \mathrm{kpc}$.
\end{abstract}

\section{Observations used}

Since the appearance of the 6-th WR Catalog (van der Hucht et al. 1981) the galactic distribution of WR stars has been studied by many authors (Hidayat et al. 1981. 1984: Meylan and Maeder, 1983: Contl et al. 1983). Recently the membership of 42 WR stars in open clusters and associations has been reassessed (Lundstrom and Stenholm. 1984) and improved photometry (Massey. 1984) and new classiflcations have become avallable for a considerable fraction of the 157 known galactic WR stars. This allows a redetermination of the intrinsic parameters $(b-v){ }_{0}$ and $M_{v}$ of $W R$ stars and their photometric distances.

\section{The WR distribution in the galactle plane}

From the distribution of their hellocentric distances we estimate that the WR star cencus is practically complete out to $d \approx 3 \mathrm{kpc}$. Considering a volume around the Sun with $d<2.5 \mathrm{kpc}$. We count 44 WR stars in the ratio $N_{W N}: N_{W C}: N_{W O}=15: 28: 1$. If we accept that the WN and WC phases are consecutive phases in the evolution of massive stars. then this implies that in the solar neighbourhood the average WC phase lifetime is twice as long as the WN phase lifetime. More specifically, we count in the range $7.5<$ $R<9.5 \mathrm{kpc}$ a WN to WC number ratio of 0.89 , and in the range $6<R<7.5 \mathrm{kpc} a$ ratio of 0.35 . The former ratio approaches the values of $t_{W N} / t_{W C}=1.22$ to 1.34 predicted by the recent evolutionary models of Maeder and Meynet (1987) for stars in the range of $M_{t}=40-60 M_{0}$ with mass loss and convective overshooting. As these authors state: the ratio $t_{W N} / t_{W C}$ is very senstitue to mass loss and overshooting. The value $N_{W N} / N_{W C}=0.35$ found in the inner galactic region compares more favourably with the evolutionary calculations for stars in the range of $M_{i}=50-60 M_{\odot}$ by Prantzos et al. (1986), who allow for more overshooting in their models than Maeder and Meynet do. and calculate $t_{W N} / t_{W C}=0.21$ to 0.34 . We confirm the strong decrease in $\mathrm{N}_{W A} / \mathrm{kpc}^{2}$ going from the inner to the outer galactic regions (a factor 9 ). but we observe no change in the WR density gradient. as suggested previously by Meylan and Maeder (1983) using the old data base of Hidayat et al. (1982). The WR density gradient is a factor 2.5 steeper than the O-type star density gradient. This could Indicate that one or more of the WR formation channels (Maeder, 1982) are relatively more effective at smaller galactocentric distances. We also confirm that the binary channel is relatively more important at larger galactocentric distances. When observed in the volume with $d<3 \mathrm{kpc}$. the WR number density can be fitted by $\log \left(\mathrm{N}_{\mathrm{WR}} / \mathrm{kpc}^{2}\right)=$ $1.96-0.21 \mathrm{R}$. with $N_{W N} / N_{W C}=0.47+0.05 R$. If this density gradient is valid throughout the Galaxy. then the total number of galactlc WR stars is about $2500<\% 1500$ WC stars). with half that number located within $3 \mathrm{kpc}$ from the galactic center.

3. WR subtype distribution and evolution

The issue of WR subtype evolution has been addressed by varlous authors in the past 
few years. In general one agrees that WC stars descend from the WN sequence and that WO stars descend from the WC sequence. but on the question how WR stars evolve from one subtype to another different ldeas have been proposed. In a very suggestive study Schild and Maeder (1984) argue on the basis of open cluster compositions for WN7 $\rightarrow$ WC4-8 evolution in young clusters. WN5-6 $\rightarrow$ WC9->WO evolution in older clusters. and WN3-5->SN evolution.

In order to Judge whether the overall galactic WR distribution poses constraints on subtype evolution. we have plotted in Fig. 1 the WR galactocentric distances $R$ (based on $R_{0}-8.5 \mathrm{kpc}$ ) versus WR subtype. This was performed earlier by Hidayat et al. (1982. Paper 1). but with the improved data base we find as more pronounced trend in Fig. 1 that the WC stars show a clear preference to have later subtypes at smaller distances from the center. WN2-5 stars are only present beyond $A \approx 6 \mathrm{kpc}$. while WN6-8 stars are present at practically all observable galactocentric distances.

Of the evolutionary road system proposed by Schild and Maeder (1984) the WN7->WC4-8 trail is accessible in Fig. 1. but there is an obstruction in their WN5 $-6 \rightarrow$ WC9 $\rightarrow$ WO trall. Fig. 1 allows the transition from WN6 to WC9. but definitely not the transition from WN5 to WC9, as was already visible in the results of Hidayat et al. (1982. Paper 1), In addition. the transition from WC9 to WO appears also excluded:

Fig. 1 allows only WC4-7 $\rightarrow$ WO evolution. In view of the alternative WC4-5p classification of the WO stars. a WC4-5 $\rightarrow$ WO trail is clearly more probable.

Fig. 1 shows that WN to WC subtype evolution is not just a function of $M_{i}$. but also of galactocentric distance. i.e. of gradients in galactlc parameters like metallicity. which in turn may reflect itself in the mass loss rates of massive stars evolving to the WR phase. Of interest in this respect is that a similar distribution as found for galactic WC subtypes has been noted recently in M31 by Moffat and Shara (1987). Also of interest is the observation by Kunth and Schild (1986) of a positive correlation between metallicity and WR star formation rate in eleven emission line galaxies. The different WN3-5 and WC4-7 distributions could support the conclusion of Schild and Maeder (1984) that WNE stars by-pass the WC phase in their further evolution. This would imply that WCE stars can only descend from WNL stars at galactocentric distances beyond $R \approx 6.5$ Kpc, and that WCL stars can only descend from WNL stars within $R \approx 8.5 \mathrm{kpc}$.

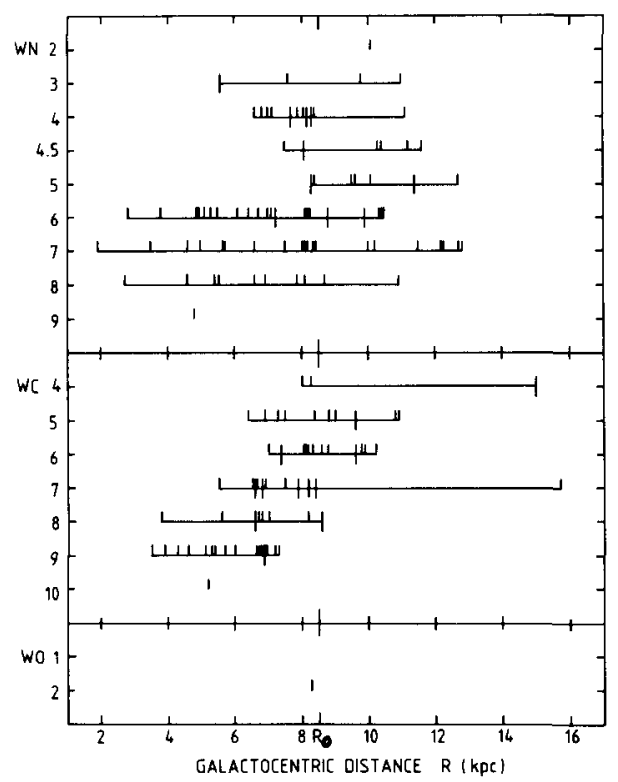

Ftgure 1. WR galactocentric distances.

\section{References}

Contl. P.S. . Garmany, C. D. . de Loore, C. . Vanbeveren. D: 1983. Astrophys. J. 274. 302. Hidayat. B. . Supelll, K. . van der Hucht. K. A. : 1982, In: C.W.H. de Loore. A.J. Willis (eds). Wolf-Rayel Stars: Observations. Physics. Evolution. Proc. IAU Symp. No. 99, p. 27. Hidayat, B. Admiranto. A. G. van der Hucht, K. A. : 1984, In: B. Hldayat. Z. Kopal. J. Raho (eds). Double Stars: Physlcal Properties and Generlc Relatlons. Proc. IAU Coll. No. 80. Astrophys. Space Sct. 99.175.

van der Hucht. K. A. . Contl. P. S. . Lundstrbm. I. . Stenholm. B. : 1981. Space Sci. Rev. 28.227. Kunth. D. Schild, H.: 1986. Astron. Astrophys, 169. 71.

Lundström. I. . Stenholm. B.: 1984c. Astron. Astrophys. Suppl. 58. 163.

Maeder. A.: 1982. Astron. Astrophys. 105. 149.

Maeder. A. . Meynet, Q.: 1987. Astron. Astrophys. 182, 243.

Massey. P.: 1984. Astrophys. J. 281. 789.

Meylan. Q. . Maeder, A.: 1983, Astron. Astrophys. 124, 84.

Moffat. A.F.J. . Shara. M. M.: 1987. Astrophys. J. 320. 266.

Prantzos. N. . Doom, C. . Arnould, M. . de Loore. C.: 1986. Astrophys. J. 304. 695.

Schlld. H., Maeder. A.: 1984. Astron. Astrophys. 136, 237. 